\title{
Maximal heat transfer between two parallel plates
}

\author{
Shingo Motoki ${ }^{1} \dagger$, Genta Kawahara ${ }^{1}$ and Masaki Shimizu ${ }^{1}$ \\ ${ }^{1}$ Graduate School of Engineering Science, Osaka University, 1-3 Machikaneyama, Toyonaka, \\ Osaka 560-8531, Japan
}

(Received xx; revised xx; accepted xx)

The divergence-free time-independent velocity vector field has been determined so as to maximise heat transfer between two parallel plates of a constant temperature difference under the constraint of fixed total enstrophy. The present variational problem is the same as that first formulated by Hassanzadeh et al. (2014); however, a search range of optimal states has been extended to a three-dimensional velocity field. The scaling of the Nusselt number $N u$ with the Péclet number $P e$ (i.e., the square root of the non-dimensionalised enstrophy with thermal diffusion timescale), $N u \sim P e^{2 / 3}$, has been found in the threedimensional optimal states, corresponding to the asymptotic scaling with the Rayleigh number $R a, N u \sim R a^{1 / 2}$, in extremely-high- $R a$ convective turbulence, and thus to the Taylor energy dissipation law in high-Reynolds-number turbulence. At $P e \sim 10^{0}$, a twodimensional array of large-scale convection rolls provides maximal heat transfer. A threedimensional optimal solution emerges from bifurcation on the two-dimensional solution branch at higher $P e$. At $P e \gtrsim 10^{3}$, the optimised velocity fields consist of convection cells with hierarchical self-similar vortical structures, and the temperature fields exhibit a logarithmic mean profile near the walls.

Key words: variational methods, mixing enhancement, Bénard convection

\section{Introduction}

What is a flow optimising heat transfer? We have explored an answer to this naive question. For buoyancy-driven convection, i.e. Rayleigh-Bénard convection, the maximal heat transfer has been discussed for more than half a century Malkus 1954; Howard 1963; Busse 1969). Kraichnan (1962) has predicted the asymptotic scaling of the Nusselt number $N u$ with the Rayleigh number $R a$ as $N u \sim R a^{1 / 2}$ with logarithmic correction for very high $R a$. In 1990's, a new variational approach called 'the background method' was invented by Doering \& Constantin (1992), and the method has triggered remarkable advancements in the theoretical estimate of the upper bound on the Nusselt number $N u$ (Doering \& Constantin 1996; Kerswell 2001; Otero et al. 2002; Plasting \& Kerswell 2003; Doering et al. 2006; Whitehead \& Doering 2011, 2012). In these theoretical works, rigorous upper bounds, e.g. $N u-1 \leqslant 0.02634 R a^{1 / 2}$ (Plasting \& Kerswell 2003), have been derived at $R a \gg 1$. The 'ultimate' law $N u \sim R a^{1 / 2}$ corresponds to the Taylor law of energy dissipation in high-Reynolds-number turbulence. It has not been demonstrated as yet what flow structure achieves the ultimate scaling $N u \sim R a^{1 / 2}$. Recently, meanwhile,

$\dagger$ Email address for correspondence: motoki@tes.me.es.osaka-u.ac.jp 


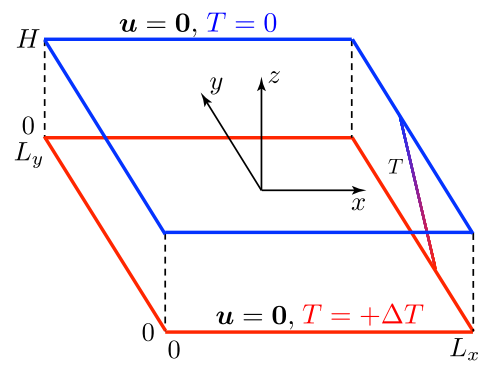

Figure 1. Configuration of the velocity and temperature fields.

Hassanzadeh et al. (2014) have numerically maximised a wall heat flux within a twodimensional velocity field bounded by two parallel plates with a constant temperature difference. They formulated a variational problem to find a velocity field maximising heat transfer under the constraint of fixed total enstrophy, and found optimal states consisting of an array of large-scale convection rolls for free-slip boundary conditions. The maximal scaling is represented by $N u \sim R a^{5 / 12}$, corresponding to the rigid upper bound derived by the background method for free-slip conditions (Whitehead \& Doering 2011, 2012). For no-slip conditions, on the other hand, the velocity fields numerically optimised within a two-dimensional field also exhibit large-scale circulation rolls, and the found scaling is $N u \sim R a^{0.37}$ (Souza 2016). Such scalings observed in the two-dimensional optimal states are quite distinct from the ultimate scaling $N u \sim R a^{1 / 2}$.

In this paper, we consider the variational problem first examined by Hassanzadeh et al. (2014) for free-slip conditions and then by Souza (2016) for no-slip conditions; however, we extend a search range of optimal states to a three-dimensional velocity field. We report three-dimensional optimal states capable of achieving the ultimate scaling, and discuss the optimised flow structures. In order to satisfy the Navier-Stokes equation the optimised divergence-free vector field needs external body force which is distinct from buoyancy, but hereafter we refer to it as a 'velocity' field.

\section{Formulation}

Let us consider heat transfer in a three-dimensional, time-independent and incompressible velocity field between two parallel plates, $\boldsymbol{u}^{\prime}\left(x^{\prime}, y^{\prime}, z^{\prime}\right)=u^{\prime} \boldsymbol{e}_{x}+v^{\prime} \boldsymbol{e}_{y}+w^{\prime} \boldsymbol{e}_{z}$, satisfying the continuity equation

$$
\nabla^{\prime} \cdot \boldsymbol{u}^{\prime}=0
$$

where a prime $(\cdot)^{\prime}$ represents a dimensional quantity, and $\boldsymbol{e}_{x}$ and $\boldsymbol{e}_{y}$ are mutually orthogonal unit vectors in the wall-parallel directions while $\boldsymbol{e}_{z}$ is a unit vector in the wall-normal direction. The configuration of the velocity and temperature fields is shown in figure 1. The two parallel plates are positioned at $z^{\prime}=0$ and $z^{\prime}=H$, and the domain of the flow is periodic in the $x$ - and $y$-directions with periods, $L_{x}^{\prime}$ and $L_{y}^{\prime}$. The upper (or lower) wall surface is held at lower (or higher) constant temperature $T^{\prime}=0$ (or $\left.T^{\prime}=\Delta T>0\right)$. We suppose that the temperature field $T^{\prime}\left(x^{\prime}, y^{\prime}, z^{\prime}\right)$ is determined as a solution to an advection-diffusion equation

$$
\left(\boldsymbol{u}^{\prime} \cdot \nabla^{\prime}\right) T^{\prime}=\kappa \nabla^{\prime 2} T^{\prime}
$$

supplemented by the boundary conditions

$$
\boldsymbol{u}^{\prime}\left(z^{\prime}=0\right)=\boldsymbol{u}^{\prime}\left(z^{\prime}=H\right)=\mathbf{0} ; \quad T^{\prime}\left(z^{\prime}=0\right)=\Delta T, \quad T^{\prime}\left(z^{\prime}=H\right)=0,
$$


where $\kappa$ denotes a thermal diffusivity. The strength of the velocity field is measured by the Péclet number $P e$ defined, in terms of the total enstrophy (or the averaged square of velocity gradient tensor), as

$$
P e=\frac{\left\langle\left|\boldsymbol{\omega}^{\prime}\right|^{2}\right\rangle^{\frac{1}{2}} H^{2}}{\kappa}=\frac{\left\langle\left|\nabla^{\prime} \boldsymbol{u}^{\prime}\right|^{2}\right\rangle^{\frac{1}{2}} H^{2}}{\kappa},
$$

where $\boldsymbol{\omega}^{\prime}=\nabla^{\prime} \times \boldsymbol{u}^{\prime},\left|\nabla^{\prime} \boldsymbol{u}^{\prime}\right|^{2}=\nabla^{\prime} \boldsymbol{u}^{\prime}: \nabla^{\prime} \boldsymbol{u}^{\prime}$ and $\langle\cdot\rangle$ is a volume average. The wall-normal convective heat transfer is characterized by the Nusselt number defined as the ratio of the convective heat flux to the conductive one,

$$
N u=1+\frac{\left\langle w^{\prime} T^{\prime}\right\rangle}{\kappa \Delta T / H} .
$$

In this study, we explore a three-dimensional velocity field maximising $N u$ for fixed $P e$. The constrained optimisation is relevant to the maximisation of the objective functional

$$
\mathcal{F}=\left\langle w \theta-\theta^{*}(\boldsymbol{x})\left[(\boldsymbol{u} \cdot \nabla) \theta-\nabla^{2} \theta-w\right]+p^{*}(\boldsymbol{x})(\nabla \cdot \boldsymbol{u})+\frac{\mu}{2}\left(P e^{2}-|\nabla \boldsymbol{u}|^{2}\right)\right\rangle
$$

(see Hassanzadeh et al. 2014), where $p^{*}(\boldsymbol{x}), \theta^{*}(\boldsymbol{x})$ and $\mu$ are Lagrange multipliers. The variables in (2.6) have been non-dimensionalised as

$$
\boldsymbol{x}=\frac{\boldsymbol{x}^{\prime}}{H}, \quad \theta=\frac{\theta^{\prime}}{\Delta T}, \quad \boldsymbol{u}=\frac{\boldsymbol{u}^{\prime}}{\kappa / H}, \quad p^{*}=\frac{p^{* \prime}}{\rho \kappa^{2} / H^{2}}, \quad \theta^{*}=\frac{\theta^{* \prime}}{\Delta T},
$$

where $\rho$ is the mass density of the fluid and $\theta=T-(1-z)$ is a temperature fluctuation about a conductive state. Stationary points of $\mathcal{F}$ are determined by the Euler-Lagrange equations

$$
\begin{aligned}
& \frac{\delta \mathcal{F}}{\delta \boldsymbol{u}} \equiv-\nabla p^{*}+\theta \nabla \theta^{*}+\mu \nabla^{2} \boldsymbol{u}+\left(\theta+\theta^{*}\right) \boldsymbol{e}_{z}=\mathbf{0}, \\
& \frac{\delta \mathcal{F}}{\delta \theta} \equiv(\boldsymbol{u} \cdot \nabla) \theta^{*}+\nabla^{2} \theta^{*}+w=0, \\
& \frac{\delta \mathcal{F}}{\delta \theta^{*}} \equiv-(\boldsymbol{u} \cdot \nabla) \theta+\nabla^{2} \theta+w=0, \\
& \frac{\delta \mathcal{F}}{\delta p^{*}} \equiv \nabla \cdot \boldsymbol{u}=0, \\
& \frac{\partial \mathcal{F}}{\partial \mu} \equiv \frac{1}{2}\left\langle P e^{2}-|\nabla \boldsymbol{u}|^{2}\right\rangle=0 .
\end{aligned}
$$

\section{Numerical optimisation}

Solutions to equations (2.8)-2.11) depend only on $\mu$ for fixed periods $\left(L_{x}, L_{y}\right)$. For given $\mu$, the solutions correspond to stationary points of the alternative functional

$$
\mathcal{G}=\left\langle w \theta-\frac{\mu}{2}|\nabla \boldsymbol{u}|^{2}-\theta^{*}(\boldsymbol{x})\left[(\boldsymbol{u} \cdot \nabla) \theta-\nabla^{2} \theta-w\right]+p^{*}(\boldsymbol{x})(\nabla \cdot \boldsymbol{u})\right\rangle .
$$

This is because $\mathcal{G}=\mathcal{F}-(\mu / 2) P e^{2}$ and thus the Euler-Lagrange equations for $\mathcal{G}$ are also given by (2.8)-(2.11). In our previous work on a different functional in a different configuration (Motoki et al. 2018), we have developed a numerical approach to find local maxima of a functional kindred to $\mathcal{G}$ by a combination of the steepest ascent method and the Newton-Krylov method. Using the same procedures, we obtain an optimal state $\left(\boldsymbol{u}_{\mathrm{opt}}, \theta_{\mathrm{opt}}, \theta_{\mathrm{opt}}^{*}, p_{\mathrm{opt}}^{*}\right)$ maximising $\mathcal{G}$ for given $\mu$. Since $\mathcal{F}$ has the gradients common to 
$\mathcal{G}$, the optimal state gives the maximum of $\mathcal{F}$ at $P e=\left\langle\left|\nabla \boldsymbol{u}_{\text {opt }}\right|^{2}\right\rangle^{1 / 2}$. Thus the optimal states of $\mathcal{F}$ can be obtained without fixing $P e$ in the process of the optimisation. Maximal points for a specific value of $P e$ (say, $\left.P e_{0}\right)$ are calculated by updating $\mu$ as

$$
\mu_{\text {new }}=\mu+\epsilon\left(\left\langle\left|\nabla \boldsymbol{u}_{\text {opt }}\right|^{2}\right\rangle-P e_{0}^{2}\right),
$$

taking account of the fact that the decrease (or increase) in $\mu$ corresponds to the increase (or decrease) in $P e$, where $\epsilon$ is a small positive constant. Equations (2.8) (2.11) are discretised employing the spectral Galerkin method based on Fourier-Chebyshev expansions (for more details, see section 3 and appendix A in Motoki et al. 2018).

In this paper, we present the optimal states in the square wall-parallel domain of $\left(L_{x}, L_{y}, L_{z}\right)=(\pi / 2, \pi / 2,1)$. The numerical computations are carried out on $64^{3}$ grid points for $\mathrm{Pe} \leqslant 5000$ and $128^{3}$ for $\mathrm{Pe}>5000$.

\section{Ultimate scaling}

Figure 2 $(a)$ shows the maximal $N u$ as a function of $P e$. At large $P e\left(>10^{3}\right)$, we observe the scaling of $N u$ with $P e, N u \sim P e^{2 / 3}$. The scaling $N u \sim P e^{2 / 3}$ corresponds to the ultimate scaling $N u \sim R a^{1 / 2}$ in the Rayleigh-Bénard problem, provided that the total energy budget is given by the Boussinesq equation, that is $P e^{2}=R a(N u-1)$ (Hassanzadeh et al. 2014), where $R a=g \beta \Delta T H^{3} /(\nu \kappa)$ is the Rayleigh number, $g$ and $\beta$ being the acceleration due to gravity and the thermal expansion coefficient of the fluid, respectively. The thick solid line indicates the rigorous upper bound derived by using the background method (Plasting \& Kerswell 2003). The obtained maximal scaling is close to the upper bound, and the prefactor is about $7.2 \%$ less than that of the bound.

Choosing the reference velocity as $U=(g \beta \Delta T H)^{1 / 2}$, we have the scaling with respect to the energy dissipation as

$$
\frac{\nu\left\langle\left|\nabla^{\prime} \boldsymbol{u}^{\prime}\right|^{2}\right\rangle}{U^{3} / H} \sim P r^{-1 / 2},
$$

where $\nu$ and $\operatorname{Pr}$ are the kinematic viscosity and the Prandtl number, respectively. Thus the scaling $N u \sim P e^{2 / 3}$ means that the energy dissipation normalised by $U^{3} / H$ is independent of the Reynolds number, in accord with the Taylor's scaling view for turbulent energy dissipation. For homogeneous turbulent convection without thermal and velocity boundary layers, e.g. in three-dimensional periodic boundary box with the vertical mean temperature gradient (Lohse \& Toschi 2003), the ultimate scaling $N u \sim R a^{1 / 2}$ has been observed. Although the Taylor dissipation law also does not hold in turbulent shear flows over a smooth wall surface, the Reynolds-number-independent skin-friction coefficient can be observed in high-Reynolds-number rough-wall turbulence, implying the emergence of the Taylor law. However, it has still been an open question whether or not the ultimate scaling can be found in high- $R a$ convective turbulence between two parallel plates with surface roughness (Roche et al. 2001; Zhu et al. 2017).

\section{Appearance of three-dimensional solution}

At large $\mu$ (small $P e$ ), a two-dimensional array of convection rolls gives maximal heat transfer. The solution arises from supercritical pitchfork bifurcation on a conductive solution at $\mu=1.703 \times 10^{-2}(P e \equiv 0)$ (figure $2 a$ ), and it satisfies the reflection symmetry

$$
[u, v, w, \theta](x, y, z)=[-u, v, w, \theta](-x, y, z)
$$


(a)

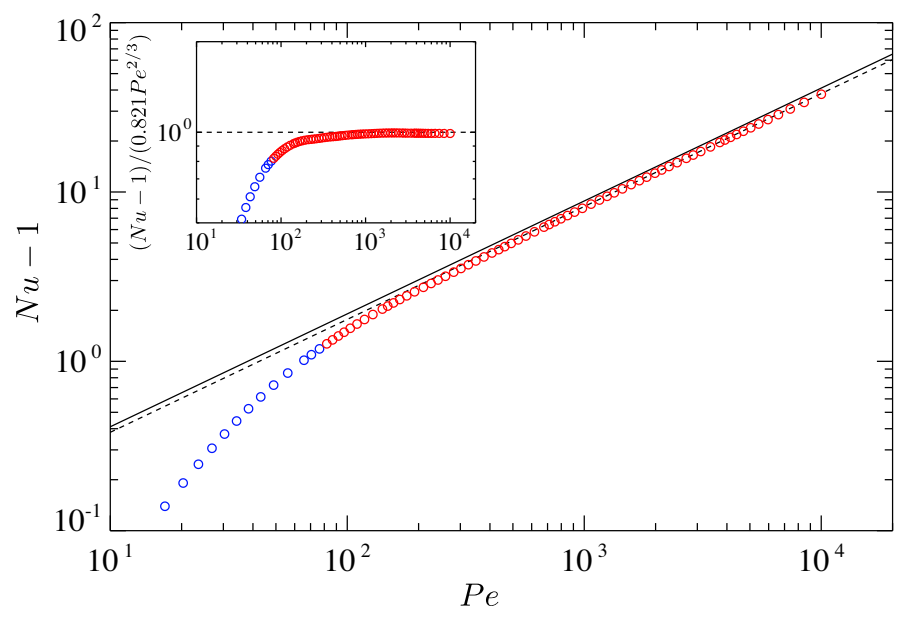

$(b)$

(c)
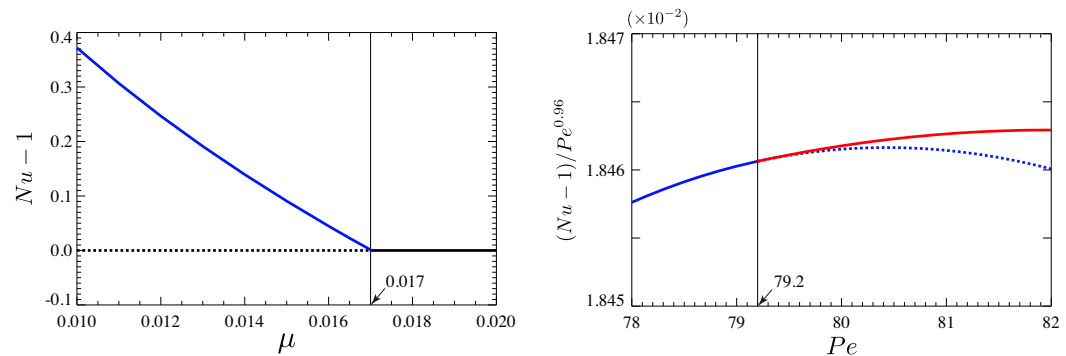

Figure 2. Nusselt number $N u$ as a function of Péclet number $P e$ in the optimal states. The blue and red circles denote two-dimensional and three-dimensional optimal states, respectively. The dashed line indicates the power fit $N u-1=0.0821 P e^{2 / 3}$ determined in the range $5 \times 10^{3}<P e<10^{4}$. The solid line represents the scaling $N u-1=0.0885 P e^{2 / 3}$ evaluated from the rigorous upper bound $N u-1=0.02634 R a^{1 / 2}$ (Plasting \& Kerswell 2003) assuming the identity $P e^{2}=R a(N u-1)$ (Hassanzadeh et al. 2014). The inset shows the compensated $N u$. $(b, c) N u$ as a function of $(b)$ much larger $\mu$ (much smaller $P e$ ) and $(c)$ larger $\mu$ (smaller $P e$ ). The blue and red curves respectively show the two-dimensional and three-dimensional solutions, and the black one is a conductive solution. The solid (or dashed) curve denotes an optimal (or saddle) solution.

and the shift-and-reflection symmetry

$$
[u, v, w, \theta](x, y, z)=[u, v,-w,-\theta]\left(x+L_{x} / 2, y, 1-z\right)
$$

(see figure 3 $a$ ). Figures $3(a, b)$ visualise isosurfaces of the temperature field $T$ and of the second invariant of the velocity gradient tensor, $Q$. As $\mu$ decreases further, the secondary pitchfork bifurcation occurs on the two-dimensional solution branch at $\mu=3.028 \times 10^{-3}$ $(P e=79.2)$ (figure 2 $c)$. Subsequently, the two-dimensional solution becomes a saddle solution, and a three-dimensional optimal solution with the shift-and-reflection symmetry

$$
[u, v, w, \theta](x, y, z)=[u, v,-w,-\theta]\left(x+L_{x} / 2, y+L_{y} / 2,1-z\right)
$$

appears (see figure $3 b$ ). Figures $3(c-e)$ show the energy spectra of the wall-normal velocity $w$ at the onset of the three-dimensional solution as a function of the distance to the wall, $z$ and the $x$-component of the wavenumber vector, $k_{x}$. In the two-dimensional 
$(a)$

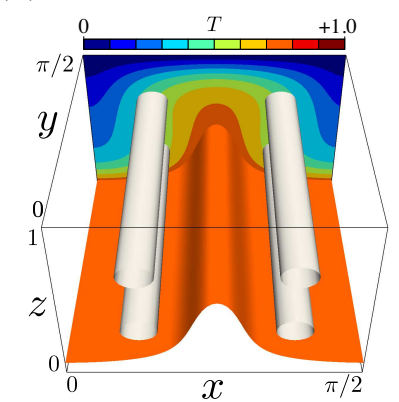

(c)

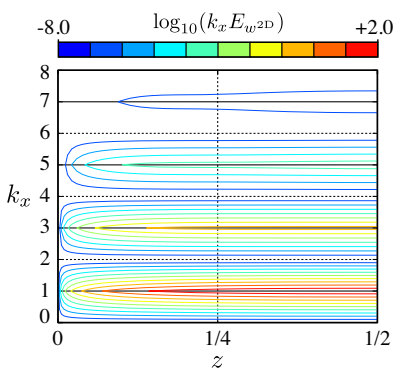

$(e)$

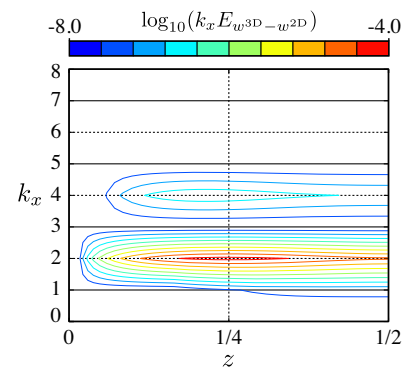

$(b)$

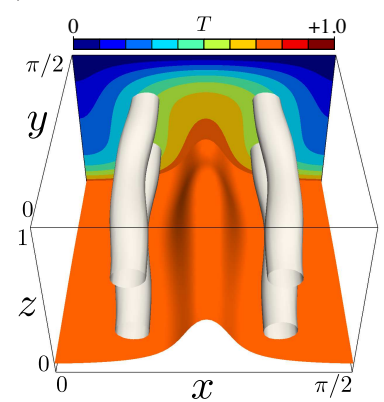

$(d)$

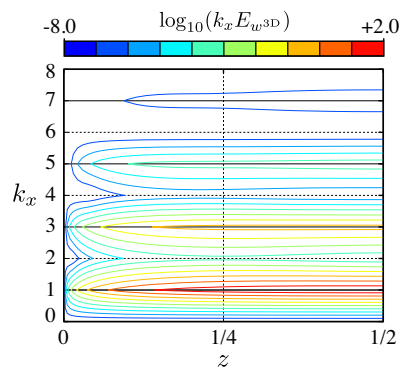

$(f)$

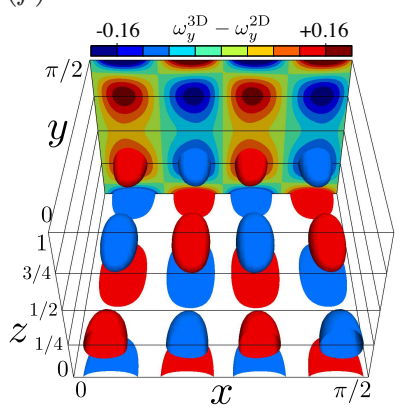

Figure 3. (a) Two-dimensional saddle and (b) three-dimensional optimal solutions at $P e=80.0$. The orange objects show the isosurfaces of the temperature, $T=0.75$, and the white tube-like objects are the vortex structures visualised by the positive second invariant of the velocity gradient tensor, $Q=2560$. The contours represent the temperature field in the plane $y=\pi / 2$. $(c-e)$ One-dimensional pre-multiplied energy spectra of the wall-normal velocity $w, k_{x} E_{w}$, at $P e=79.2$. The spectrum of $(c)$ the two-dimensional solution $w^{2 \mathrm{D}}$ and $(d)$ the three-dimensional solution $w^{3 \mathrm{D}}$; and of $(e)$ their difference $w^{3 \mathrm{D}}-w^{2 \mathrm{D}}$. The lateral axis denotes the distance to the wall $z$, and the longitudinal one is the wavenumber component $k_{x}$ in the $x$-direction. $(f)$ Spatial distribution of the difference in the $y$-component of vorticity $\omega_{y}$ between the three- and two-dimensional solutions, $\omega_{y}^{3 \mathrm{D}}-\omega_{y}^{2 \mathrm{D}}$ at $P e=79.2$. The red/blue objects respectively show the isosurfaces of $\omega_{y}^{3 \mathrm{D}}-\omega_{y}^{2 \mathrm{D}}= \pm 0.12$. The contours represent $\omega_{y}^{3 \mathrm{D}}-\omega_{y}^{2 \mathrm{D}}$ in the plane $y=\pi / 2$.

solution shown in figure $3(c)$, the wall-normal velocity $w$ consists of only odd-wavenumber components. The spectral peak is located for $k_{x}=1$ at the midplane $z=1 / 2$, and it is relevant to the large-scale rolls. The even-wavenumber components appear as a result of the bifurcation from the two-dimensional solution to the three-dimensional one (figure [3d). The difference in the spectra between the two solutions at $P e=79.2$ is shown in figure $3(e)$. The leading mode is at $k_{x}=2$, and the spectral component has a peak at 
$z=1 / 4$ (half the distance between one of the two walls and the midplane). In figure $3(f)$, the relevant structures are visualised by the difference in the $y$-component of vorticity, $\omega_{y}^{3 \mathrm{D}}-\omega_{y}^{2 \mathrm{D}}$. The extracted structure is characterised in terms of a three-dimensional mode $\left(k_{x}, k_{y}\right)=(2,1)$, and exhibits an array of vortices arranged in a wall-parallel plane around $z=1 / 4$. The onset of the smaller three-dimensional vortical structures near the walls brings about the bending of the original larger two-dimensional rolls and associated vortex tubes (figure $3 b$ ), enhancing heat transfer.

\section{Hierarchical self-similar structures}

Tree-like structure of isotherms is observed in the optimal states at small $\mu$ (large $P e$ ), shown in figures 4 . The orange objects show isosurfaces of $T=0.75$, and a 'trunk' of the 'tree' represents a hot 'plume' where the positive wall-normal velocity has been found to be dominant. As $P e$ increases, the tree 'roots' grow deeper while maintaining the large-scale trunk. The white objects show smallest-scale vortex structures visualised by the positive iso-surfaces of the second invariant of the velocity gradient tensor in the near-wall region of the lower half of the domain (similar vortical structures exist on the upper wall). The smaller and stronger vortices appear closer to the walls with increasing the enstrophy, i.e. Pe. The roots are seen to be generated as a consequence of upward fluid motion induced in between the roughly anti-parallel nearest segments of the winding tube-like vortices. As seen in the bifurcation of the three-dimensional solution from the two-dimensional solution, the local folding of the larger vortices stems from the onset of the smaller vortical structures closer to the wall. Figure 5 shows the energy spectra of the wall-normal velocity $w$ as a function of the distance to the wall, $z$ and the wavelength in the $x$-direction, $\lambda_{x}=L_{x} / k_{x}$ relevant to the size of the vortical structures. It can be seen that smaller-scale structures are generated closer to the wall as $P e$ is increased. At $P e=10009$ several spectral peaks are observed along the 'ridge' represented by the dashed diagonal $\lambda_{x}=L_{x} z$, implying that the optimal velocity fields possess hierarchical self-similarity. As shown in figure 6(a), the energy spectra scale with the conduction length $\lambda_{\theta}=(2 N u)^{-1}$ in the close vicinity of the wall. The hierarchical structures exist down to $z / \lambda_{\theta} \approx 1$, where the size of the structures is $\lambda_{x} \approx 5 N u^{-1}$. Since $N u$ scales with $P e^{2 / 3}$ at large $P e$, the smallest scale is estimated as $\lambda_{x} \sim P e^{-2 / 3}$ much smaller than the optimal aspect ratio, $L / H \sim P e^{-0.371}$, in the two-dimensional field (Souza 2016). Figure $6(b)$ shows the mean temperature profile $\bar{T}$ as a function of $z / \lambda_{\theta} \cdot 1-\bar{T}=z / \lambda_{\theta}$ holds at $z / \lambda_{\theta} \ll 1$, where the thermal conduction dominates over the convection. As the distance to the wall, $z$ increases, the hierarchical vortex structures promote the convective heat transfer. In the region $1 \lesssim z / \lambda_{\theta} \lesssim 10$, the logarithmic-like temperature profiles are observed at $P e=1008,5041$ and 10009. The dashed line indicates the logarithmic fit $1-\bar{T}=0.0358 \ln \left(z / \lambda_{\theta}\right)+0.423$ determined in the range $2<z / \lambda_{\theta}<4$ at $P e=10009$. Recently, the logarithmic temperature profiles have also been observed numerically and experimentally in turbulent Rayleigh-Bénard convection (Ahlers et al. 2012, 2014). In the region far from the wall, $10 \lambda_{\theta} \lesssim z \leqslant 1 / 2$, mixing by the large-scale convection cells is dominant, and thus the temperature profile is flattened.

\section{Summary and conclusions}

We have found the three-dimensional optimal states which lead to the scaling $N u \sim$ $P e^{2 / 3}$ consistent with the ultimate scaling $N u \sim R a^{1 / 2}$ in Rayleigh-Bénard convection. The optimal heat transfer is achieved by three-dimensional convection cells with smallerscale vortices attached on the walls. At large $P e$, the optimal velocity field exhibits 
$(a)$

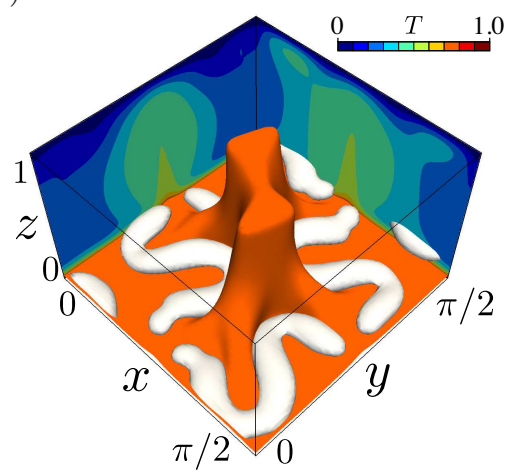

(c)

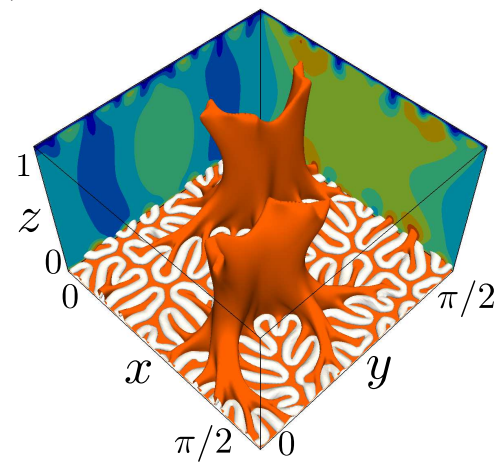

(b)

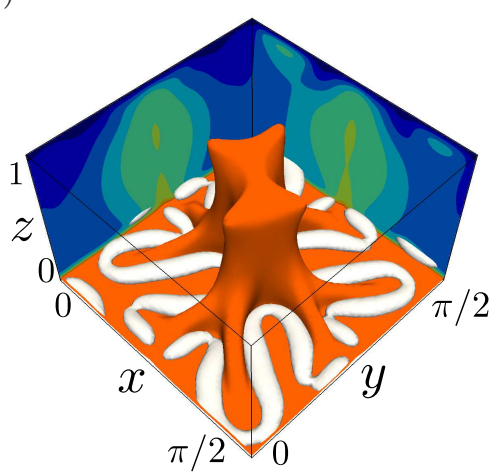

(d)

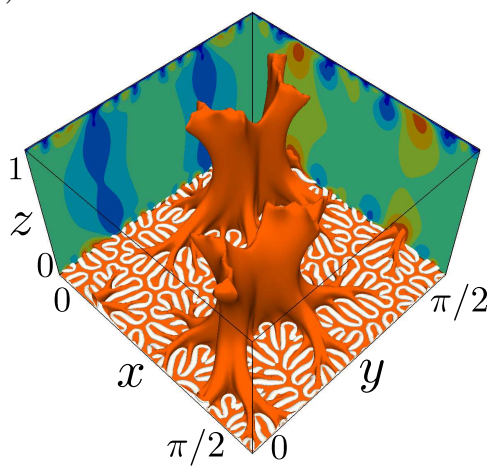

Figure 4. Optimal states at Péclet number (a) $P e=508$, (b) $P e=1006$, (c) $P e=5041$ and (d) $P e=10009$. The orange objects show the isosurfaces of $T=0.75$. The white tube-like structures are the isosurfaces of $(a) Q=8.0 \times 10^{4},(b) Q=4.8 \times 10^{4},(c) Q=1.6 \times 10^{7}$ and $(d)$ $Q=1.6 \times 10^{8}$ (note that only those in the lower half of the domain are shown for visualisation of the near-wall structures). The contours represent temperature field in the planes $x=\pi / 2$ and $y=0$.

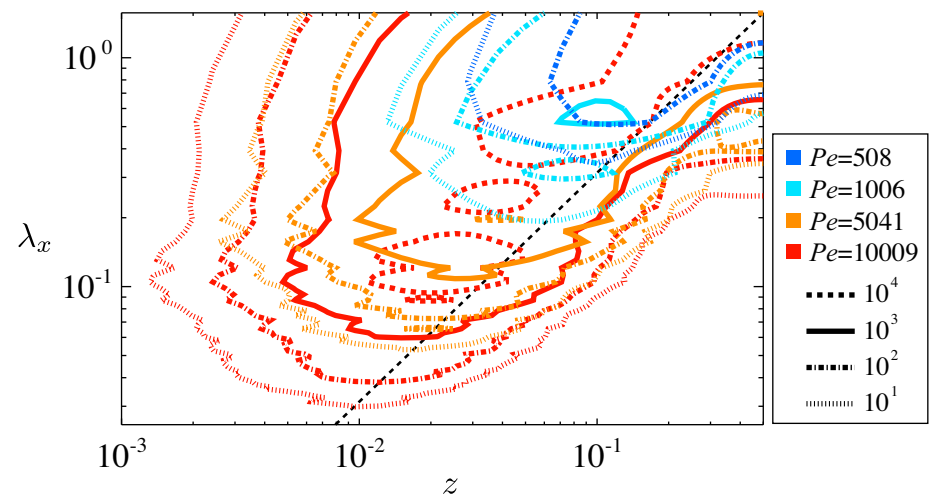

FiguRE 5. Energy spectra of the wall-normal velocity $w, k_{x} E_{w}$, as a function of the distance to the wall, $z$ and the wavelength in the $x$-direction, $\lambda_{x}$. The dashed diagonal indicates $\lambda_{x}=L_{x} z$. 
(a)

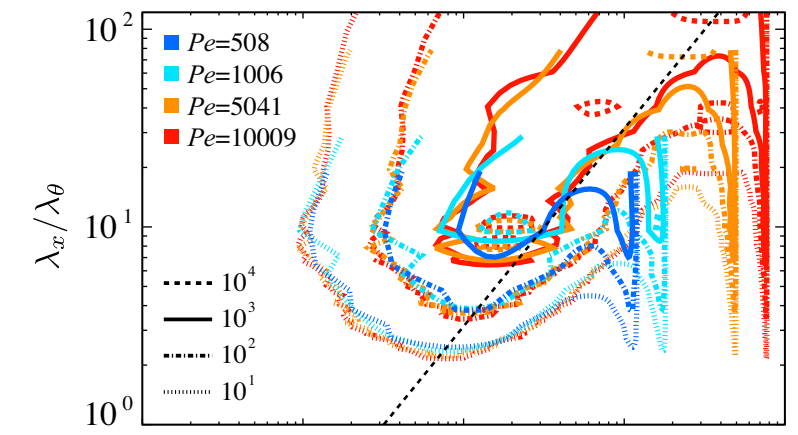

(b)

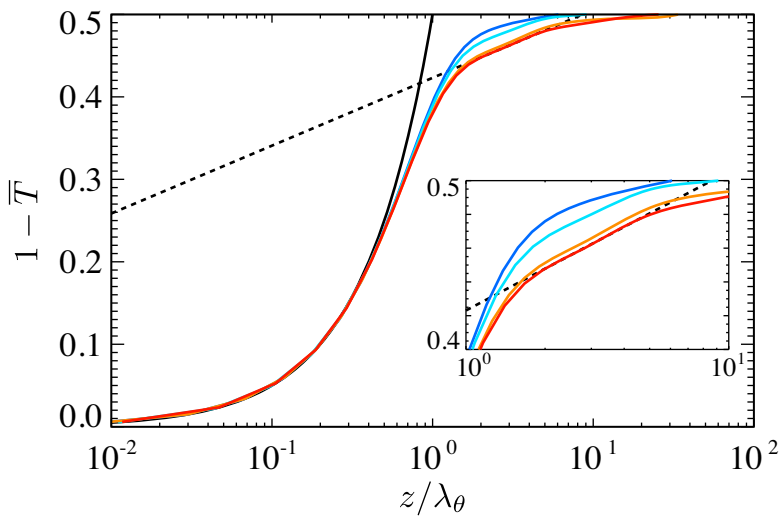

FiguRE 6. (a) Energy spectra $k_{x} E_{w}$ as a function of $z$ and $\lambda_{x}$. The distance to the wall, $z$ and the wavelength in the $x$-direction, $\lambda_{x}$ are normalised by $\lambda_{\theta}=(2 N u)^{-1}$. The dashed diagonal indicates $\lambda_{x}=L_{x} z$. (b) Mean temperature profile $\bar{T}$ as a function of $z / \lambda_{\theta}$. The solid curve indicates $1-\bar{T}=z / \lambda_{\theta}$, and the dashed line represents the logarithmic fit $1-\bar{T}=0.0358 \ln \left(z / \lambda_{\theta}\right)+0.423$ determined in the range $2<z / \lambda_{\theta}<4$ at $P e=10009$.

hierarchical self-similarity. The large-scale cells mix up the temperature almost completely around the midplane between the two walls. Near the walls, meanwhile, selfsimilar vortical structures locally enhance heat transfer, and yield logarithmic mean temperature distribution. Our earlier optimisation for heat transfer in plane Couette flow (Motoki et al. 2018) provided the optimal velocity fields in which we observed hierarchical structure consisting of a number of streamwise vortex tubes. The logarithmic mean temperature profiles as well as the ultimate scaling $N u \sim R a^{1 / 2}$ were also found in the optimal fields. It has recently been observed that the ultimate scaling $N u \sim R a^{1 / 2}$ can be achieved by some velocity field which is two-dimensional but exhibits hierarchical selfsimilarity (Tobasco \& Doering 2017). These results suggest that self-similar hierarchy of a velocity field would be a necessary condition for the emergence of the ultimate scaling and logarithmic mean temperature profile between two-parallel no-slip plates. The optimal state for heat transfer identified in this work should be closely relevant to convective turbulence, although external body force to be necessary for the optimal state to fulfill the Navier-Stokes equation is different from buoyant force in the Boussinesq equation. Our preliminary study, in reality, demonstrates that by using homotopy from the body force to the buoyancy the optimal state can be continuously connected to 
a steady solution to the Boussinesq equation, which well represents the structure and statistics of convective turbulence.

\section{Acknowledgements}

This work was partially supported by a Grant-in-Aid Scientific Research (grant nos. 25249014, 26630055) from the Japanese Society for Promotion of Science 665 (JSPS). S.M. is supported by JSPS Grant-in-Aid for JSPS Fellows Grant Number 666 16J00685.

\section{REFERENCES}

Ahlers, G., Bodenschatz, E., Funfschilling, D., Grossmann, S., He, X., Lohse, D., Stevens, R. J. A. M. \& Verzicco, R. 2012 Logarithmic temperature profiles in turbulent Rayleigh-Bénard convection. Phys. Rev. Lett. 109, 114501.

Ahlers, G., Bodenschatz, E. \& He, X. 2014 Logarithmic temperature profiles of turbulent Rayleigh-Bénard convection in the classical and ultimate state for a Prandtl number of 0.8. J. Fluid Mech. 758, 436-467.

Busse, F. H. 1969 On Howard's upper bound for heat transport by turbulent convection. J. Fluid Mech. 37, 457-477.

Doering, C. R. \& Constantin, P. 1992 Energy dissipation in shear driven turbulence. Phys. Rev. Lett. 69, 1648-1651.

Doering, C. R. \& Constantin, P. 1996 Variational bounds on energy dissipation in incompressible flows. III. Convection. Phys. Rev. E 53 (6), 5957-5981.

Doering, C. R., Otto, F. \& Reznikoff, M. G. 2006 Bounds on vertical heat transport for infinite-Prandtl-number Rayleigh-Bénard convection. J. Fluid Mech. 560, 229-241.

Hassanzadeh, P., Chini, G. P. \& Doering, C. R. 2014 Wall to wall optimal transport. J. Fluid Mech. 751, 627-662.

Howard, L. N. 1963 Heat transport by turbulent convection. J. Fluid Mech. 17, 405-432.

Kerswell, R. R. 2001 New results in the variational approach to turbulent Boussinesq convection. Phys. Fluids 13, 192-209.

Kraichnan, R. H. 1962 Turbulent thermal convection at arbitrary Prandtl number. Phys. Fluids 5 (1374).

Lohse, D. \& Toschi, F. 2003 Ultimate state of thermal convection. Phys. Rev. Lett. 90 (034502).

Malkus, W. V. R. 1954 The heat transport and spectrum of thermal turbulence. Proc. R. Soc. Lond. A 225, 196-212.

Motoki, S., Kawahara, G. \& Shimizu, M. 2018 Optimal heat transfer enhancement in plane Couette flow. J. Fluid Mech. 835, 1157-1198.

Otero, J., Wittenberg, R. W., Worthing, R. A. \& Doering, C. R. 2002 Bounds on Rayleigh-Bénard convection with an imposed heat flux. J. Fluid Mech. 473, 191-199.

Plasting, S. C. \& Kerswell, R. R. 2003 Improved upper bound on the energy dissipation rate in plane Couette flow: the full solution to Busse's problem and the Constantin-DoeringHopf problem with one-dimensional background field. J. Fluid Mech. 477, 363-379.

Roche, P. E., Castaing, B., Chabaud, B. \& Hébral, B. 2001 Observation of the $\frac{1}{2}$ power law in Rayleigh-Bénard convection. Phys. Rev. E 63 (045303(R)).

SouzA, A. N. 2016 An optimal control approach to bounding transport properties of thermal convection. Ph.D. thesis, University of Michigan .

Tobasco, I. \& Doering, C. R. 2017 Optimal wall-to-wall transport by incompressible flows. Phys. Rev. Lett. 118, 264502.

Whitehead, J. P. \& Doering, C. R. 2011 Ultimate state of two-dimensional Rayleigh-Bénard convection between free-slip fixed-temperature boundaries. Phys. Rev. Lett. 106 (244501).

Whitehead, J. P. \& Doering, C. R. 2012 Rigid bounds on heat transport by a fluid between slippery boundaries. J. Fluid Mech. 707, 241-259.

Zhu, X., Stevens, R. J. A. M., Verzicco, R. \& Lohse, D. 2017 Roughness-facilitated local $1 / 2$ scaling does not imply the onset of the ultimate regime of thermal convection. Phys. Rev. Lett. 119 (154501). 